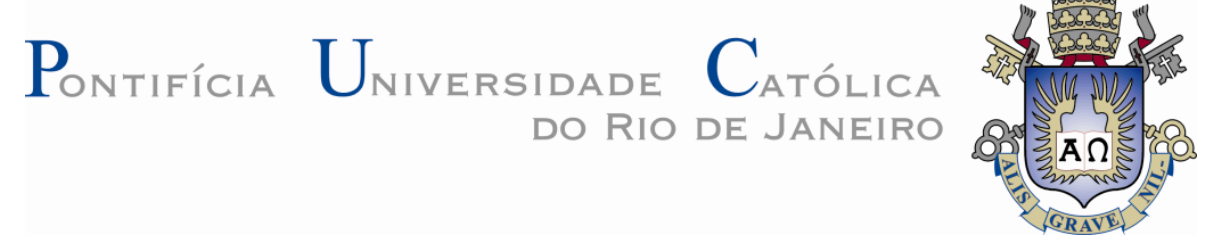

Claudio Vidal Teixeira

\title{
Ignição por Compressão com Reatividade Controlada e Dupla Injeção Direta Diesel-Etanol
}

Tese apresentada ao Programa de Pós-graduação em Engenharia Mecânica da PUC-Rio como requisito parcial para obtenção do grau de Doutor em Engenharia Mecânica.

Orientador: Prof. Sergio Leal Braga Co-Orientador: Prof. Carlos Valois Maciel Braga 


\section{Pontifícia Universidade Católica $_{\text {a }}$

Claudio Vidal Teixeira

\section{Ignição por Compressão com Reatividade Controlada \\ e Dupla Injeção Direta Diesel-Etanol}

Tese apresentada como requisito parcial para obtenção do grau de Doutor pelo Programa de Pós-Graduação em Engenharia Mecânica da PUC-Rio. Aprovada pela Comissão Examinadora abaixo assinada.

Prof. Sergio Leal Braga

Orientador

Departamento de Engenharia Mecânica - PUC-Rio

Prof. Carlos Valois Maciel Braga

Coorientador

Departamento de Engenharia Mecânica - PUC-Rio

Prof. Jose Alberto dos Reis Parise

Departamento de Engenharia Mecânica - PUC-Rio

Dr. Fernando Zegarra Sánchez

Laboratório de Engenhara Veicular - PUC-Rio

Prof. Juan Jose Milon Guzman

Universidad Tecnológica del Perú

Dr. Antônio Carlos Scardini Villela

Petrobras

Prof. Victor Santoro Santiago

Departamento de Engenharia Mecânica - IME

Prof. Márcio da Silveira Carvalho

Coordenador Setorial do Centro

Técnico Científico - PUC-Rio

Rio de Janeiro, 31 de outubro de 2018 
Todos os direitos reservados. É proibida a reprodução total ou parcial do trabalho sem autorização da universidade, do autor e do orientador.

\section{Claudio Vidal Teixeira}

Graduou-se em Engenharia Mecânica e de Automóveis no Instituto Militar de Engenharia (IME), em novembro de 2004. De 2004 a 2008, atuou como engenheiro de manutenção de viaturas e equipamentos no $3^{\circ}$ Batalhão de Engenharia de Construção ( $3^{\circ}$ BECnst) do Exército Brasileiro. Obteve o Título de Mestre em Engenharia Mecânica no IME, em abril de 2010. De 2010 a 2013, desempenhou, no Centro Tecnológico do Exército (CTEx), a função de gerente de projeto da Viatura Leve de Emprego Geral Aerotransportável (VLEGA GAUCHO). Atualmente desempenha, no Diretoria de Fabricação (DF) do Exército Brasileiro, a função de fiscal de contrato do projeto Viatura Blindada Transporte de Pessoal - Média de Rodas Guarani (VBTP-GUARANI).

Ficha Catalográfica

Teixeira, Claudio Vidal

Ignição por compressão com reatividade controlada e dupla injeção direta diesel-etanol / Claudio Vidal Teixeira; orientador: Sergio Leal Braga; co-orientador: Carlos Valois Maciel Braga. - 2018.

144 f.: il. color.; $30 \mathrm{~cm}$

Tese (doutorado) - Pontifícia Universidade Católica do Rio de Janeiro, Departamento de Engenharia Mecânica, 2018.

Inclui bibliografia

1. Engenharia Mecânica - Teses. 2. Ignição por compressão com reatividade controlada. 3. RCCI. 4. Diesel. 5. Etanol. I. Braga, Sergio Leal. II. Braga, Carlos Valois Maciel. III. Pontifícia Universidade Católica do Rio de Janeiro. Departamento de Engenharia Mecânica. IV. Título. 


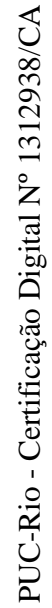

Para os meus pais, Manoel e Maria, pelo amor incondicional. 


\section{Agradecimentos}

A Pontifícia Universidade Católica do Rio de Janeiro (PUC-Rio), ao Departamento de Engenharia Mecânica da PUC-Rio (DEM) e ao Instituto de Tecnologia da PUC-Rio (ITUC) que me receberam como doutorando e proporcionaram as condições adequadas para a realização deste trabalho.

Ao Exército Brasileiro, ao Instituto Militar de Engenharia (IME) ao Centro Tecnológico do Exército (CTEx), Ao Arsenal de Guerra do Rio de Janeiro (AGR) e a Diretoria de Fabricação (DF) pelo apoio irrepreensível que me franquiaram durante o doutorado.

Aos Professores Sergio Leal Braga e Carlos Valois Maciel Braga que, foram mais do que orientadores. Foram fiadores de um trabalho experimental cujas dificuldades apontavam para o fracasso. Mesmo assim não desistiram, permaneceram comigo e confiaram no sucesso do trabalho.

Ao Professor Jose Alberto dos Reis Parise que pelos aconselhamentos. Foram determinantes para que continuasse persistindo em alcançar o objetivo.

Ao Doutor Fernando Zegarra Sánchez que acompanhou o trabalho desde o início. E, mesmo repleto de tarefas, colaborou efetivamente em todos os setores: montagem do experimento, aquisição e reparo de componentes, operação dos testes e do programa Matlab e na orientação na escolha dos parâmetros para composição dos testes.

Aos integrantes do ITUC, Marcos, Renato, Leandro, Geovani, Janaina, Renata, Florian, Luciano, pela dedicação e fidalguia empregada no trato com o corpo discente, do qual fiz parte por alguns anos. 
Aos integrantes do LEV, Bruno, Jorge, Gerson, Gilson, Leonardo, Nestor, Severino, Pedro Paulo e Paulo Sergio, pela infinita paciência e empenho exemplar. Sem esses personagens o trabalho experimental sequer iniciaria.

Aos Professores Juan Jose Milon Guzman (UTP) e Victor Santoro Santiago (IME) e ao Doutor Antônio Carlos Scardini Villela (PETROBRAS) que reservaram parte do precioso tempo para fazer parte da banca.

Ao meu tutor no IME, Professor Francesco Scofano Neto, que acompanhou todas as fases do doutorado e se engajou pessoalmente nas demandas e solicitações de prorrogação. Importantíssimo. Sem as prorrogações não seria possível terminar o trabalho.

Ao General Prado e ao Coronel Armando, respectivamente, comandante e subcomandante do IME, que acreditaram no meu trabalho e fizeram gestões para que eu pudesse comparecer a PUC Rio e terminar os experimentos, mesmo depois ter sido transferido do IME.

Ao General Luiz Henrique e ao Coronel Correia, respectivamente, comandante e subcomandante da DF, que depositaram confiança em mim e proporcionaram as condições apropriadas para continuar empenhado na tese de doutorado, simultaneamente aos serviços da caserna sob minha responsabilidade.

Ao Coronel Rubens, Coronel Samir, Coronel Loriato, todos da DF, que compreenderam a minha condição de doutorando e me apoiaram em todas as circunstâncias.

Ao General Robson, Coronel Ademir, Tenente Coronel Marcio Gomes e Tenente Coronel Inácio, Servidor Civil Evandro e Servidora Civil Zuleica, todos do CTEx, pela empatia, compreensão e apoio. Estiveram presentes e atuantes no momento mais difícil do doutorado.

Ao Tenente Lopo, Subtenente Gonçalves, Subtenente Ferreira, Sargento Artur, Sargento Valdir, Sargento Alexander, Servidor Civil Ferreira e Servidor Civil Alexandre, Servidor Civil Andreaze e Servidor Civil Lincon, todos do CTEx pela imensa paciência, dedicação e desapego com o horário de almoço. O empenho de parte desse período de descanso tornou possível finalizar os desenhos dos alojamentos dos injetores de diesel e de etanol no cabeçote do motor, recalcular a nova taxa de compressão, confeccionar os alojamentos dos injetores, modificar o 
cabeçote para receber os injetores, adaptar o sistema de injeção de etanol, além dos inúmeros serviços de reparação relacionados à bancada experimental.

Ao Wagner Santaniello da YANMAR SOUTH AMERICA por ter gentilmente cedido um cabeçote do motor Yanmar NSB50 para ser adaptado e funcionar com tecnologia RCCI. E por ter disponibilizado tempo para de sanar as dúvidas relacionadas aos aspectos dimensionais do motor.

Ao Felipe da PWM SOFTWARE, que ultrapassou as fronteiras da prestação de serviço e se empenhou com esmero nos projetos de controle dos sistemas de injeção de etanol e diesel, inclusive nos finais de semana e feriados.

Ao engenheiro Mario da MARONI Ltda., por ter aceito o desafio de reparar os componentes da bancada experimental, empregando toda experiência e genialidade para desempenhar o serviço em prazos curtíssimos.

Aos meus irmãos da igreja, que torceram por mim se dispuseram a jejuar e a interceder em oração pelo meu sucesso.

Aos meus irmãos biológicos, Clea e Cleiton, que acompanharam a minha trajetória, oraram e compartilharam dos diversos sentimentos presentes em um doutorado. Muito obrigado pelo apoio.

Aos meus filhos, Mylena Muniz Vidal Teixeira e Leonardo Muniz Vidal Teixeira, que me alegraram e me inspiraram, principalmente, nas adversidades. Inocentemente, transportaram-me para a real dimensão da vida e me fizeram lembrar de um ensinamento básico: com saúde é fácil ser feliz.

À minha esposa, Andrea dos Santos Muniz da Silva, pela compreensão e pelo apoio incondicional, sem os quais não seria possível continuar.

Aos meus pais Manoel Teixeira Lima e Maria Vidal Teixeira, que dedicaram a vida à família. E conseguiram com amor e simplicidade transmitir ensinamentos sólidos, deixando como herança o maior legado que os filhos podem ter: a educação.

A Deus, pelo milagre. Por ter me presenteado com a força de trabalho dos melhores recursos humanos disponíveis no Brasil e com a possibilidade de usufruir de Instituições tradicionais e respeitadas no campo científico internacional.

O presente trabalho foi realizado com apoio da Coordenação de Aperfeiçoamento de Pessoal de Nível Superior - Brasil (CAPES) - Código de Financiamento 001. 


\section{Resumo}

Teixeira, Claudio Vidal; Braga, Sergio Leal; Braga, Carlos Valois Maciel. Ignição por compressão com reatividade controlada e dupla injeção direta Diesel-Etanol. Rio de Janeiro, 2018. 144p. Tese de Doutorado Departamento de Engenharia Mecânica, Pontifícia Unversidade Católica do Rio e Janeiro.

Uma tecnologia desenvolvida na Universidade de Wisconsin-Madison denominada de "Reactivity Controlled Compression Ignition (RCCI)" usa dois injetores, por cilindro, para misturar combustível de baixa-reação (gasolina) com combustível de alta-reação (diesel) em um motor de ignição por compressão (ICO). Esta técnica possibilitou maior controle do processo de combustão, diminuição do consumo de combustível e dos gases de exaustão prejudiciais ao meio ambiente.Neste trabalho foi utilizado um motor ICO monocilíndrico, modificado para operar com tecnologia RCCI, injetando diesel e etanol diretamente na câmara de combustão. O objetivo era alcançar a maior taxa de substituição de diesel por etanol, utilizando estratégias de dupla e tripla injeção de combustível. Os resultados dos testes mostram que, operando com a estratégia de dupla injeção de combustível (etanol à $-170^{\circ}$ PMS e diesel a $-8^{\circ}$ PMS), a eficiência do motor modificado melhorou, mas surgiram pontos de alta pressão no interior do cilindro capazes de danificar o motor. Utilizando outra estratégia de dupla injeção de combustível (diesel a $-8^{\circ}$ PMS e etanol à $+4^{\circ}$ PMS) não foram constatados pontos de alta pressão no interior do cilindro, mas ocorreu um decréscimo na eficiência. Os resultados mais promissores foram obtidos empregando a estratégia de tripla injeção de combustível (etanol à $-170^{\circ} \mathrm{PMS}$, diesel a $-8^{\circ} \mathrm{PMS}$ e etanol à $+4^{\circ} \mathrm{PMS}$ ): a eficiência aumentou e foi alcançada a maior taxa de substituição de diesel por etanol (74,6\%).

\section{Palavras chave}

Ignição por Compressão com Reatividade Controlada; RCCI; Diesel; Etanol. 


\section{Abstract}

Teixeira, Claudio Vidal; Braga, Sergio Leal (Advisor); Braga, Carlos Valois Maciel (Coadvisor). Reactivity controlled compresion ignition with double direct injection Diesel-Ethanol. Rio de Janeiro, 2018. 144p. Tese de Doutorado - Departamento de Engenharia Mecânica, Pontifícia Unversidade Católica do Rio e Janeiro.

A technology developed at the University of Wisconsin-Madison called "Reactivity Controlled Compression Ignition (RCCI)" uses two injectors, per cylinder, to mix low-reaction fuel (gasoline) with high-reaction (diesel) fuel ignition (ICO). This technique allowed greater control of the combustion process, reduction of fuel consumption and exhaust gases harmful to the environment. In this work was used a single-cylinder compression ignition (IC) engine, modified to operate with RCCI technology, injecting diesel and ethanol directly into the combustion chamber. The objective was to achieve the highest rate of substitution of diesel by ethanol, using double and triple fuel injection strategies. Test results show that modified engine efficiency improved when the dual fuel injection strategy (ethanol at $-170^{\circ} \mathrm{PMS}$ and diesel at $-8^{\circ} \mathrm{PMS}$ ) was used, but high pressure points appeared inside the cylinder that could damage the engine. Using another dual fuel injection strategy (diesel at $-8^{\circ} \mathrm{PMS}$ and ethanol at $+4^{\circ} \mathrm{PMS}$ ) no pressure peaks were detected inside the cylinder, but a decrease in efficiency occurred. The most promising results were obtained using the triple fuel injection strategy (ethanol at $-170{ }^{\circ} \mathrm{PMS}$, diesel at $-8^{\circ} \mathrm{PMS}$ and ethanol at $\left.+4{ }^{\circ} \mathrm{PMS}\right)$ : efficiency increased and the highest diesel substitution rate by ethanol was achieve $(74,6 \%)$.

\section{Keywords}

Compression Ignition Controlled Reactivity; RCCI; Diesel; Ethanol. 


\section{Sumário}

1. Introdução 22

1.1. Tecnologia $\mathrm{RCCl} 25$

1.2. Objetivos 27

1.3. Contribuição 28

1.4. Organização da Tese 28

2. Revisão Bibliográfica 29

2.1 Pesquisas relacionadas à Tecnologia $\mathrm{RCCl} \quad 31$

3. Descrição dos Equipamentos 38

3.1 Bancada Experimental do Motor Original 38

3.2 Bancada Experimental do Motor Modificado 39

3.3 Motor Diesel $\quad 40$

3.4 Conjunto Roda Fônica - Sensor de posição 41

3.4.1 Roda Fônica 42

3.4.2 Sensor de Posição 42

3.5 Bancada Dinamométrica 43

3.5.1 Sensor de Torque 43

3.5.2 Inversor de Frequência 44

3.5.3 Dissipador de Potência 44

3.5.4 Motor Elétrico do Dinamômetro 45

3.6 Sensor de Pressão $\quad 45$

3.7 Sensor de Vazão de Combustível 46

3.8 Sensor de Vazão de $\operatorname{Ar} \quad 47$

3.9 Balança Sartorius PMA7501 48

3.10 Sistema de Injeção de Etanol (SIE) 48

3.10.1 Subsistema de Baixa Pressão 50

3.10.2 Bomba de Combustível de Alta Pressão 51

3.10.3 Mangueira de Combustível de Alta Pressão e Acumulador 51

3.10.4 Common Rail - Etanol 52

3.10.5 Injetor de Etanol 52 
3.10.6 Inversor de Frequência do Sistema de Injeção de Etanol 53

3.10.7 Motor Elétrico do Sistema de Injeção de Etanol 54

3.10.8 Fonte Estabilizada do Sistema de Injeção de Etanol 54

3.11 Sistema Lubrificante da Unidade de Acionamento 55

3.12 Sistema de Injeção de Diesel (SID) 55

3.12.1 Bomba de Alta Pressão - Diesel 56

3.12.2 Common Rail - Diesel 57

3.12.3 Injetor - Diesel 58

3.12.4 Motor Elétrico do Sistema de Injeção de Diesel 58

3.13 Cabeçote Modificado 58

4. Metodologia 64

4.1 Primeira Etapa dos Experimentos 65

4.1.1 Determinação dos Parâmetros de Funcionamento do Motor Original YANMAR NSB50 - Teste 0

4.1.2 Mapeamento dos injetores (Etanol e Diesel) 66

4.1.3 Mapeamento do motor modificado - Teste D 68

4.1.3.1 Teste D1 68

4.1.3.2 Teste D2 68

4.1.3.2 Teste D3 69

4.2 Segunda Etapa dos Experimentos $\quad 70$

4.2.1 Teste DE: Motor Modificado Operando com Dupla Injeção (Diesel / Etanol) 70

4.2.2 Teste ED: Motor Modificado Operando com Dupla Injeção (Etanol / Diesel) 71

4.2.3 Teste EDE: Motor Modificado Operando com Tripla Injeção (Etanol / Diesel / Etanol)

5 Tratamento dos Dados 73

5.1 Potência Efetiva $\quad 74$

5.2 Consumo Específico de Combustível $\quad 74$

5.3 Rendimento Térmico $\quad 75$

5.4 Vazão Mássica de Ar Seco $\quad 76$

5.4.1 Vazão Volumétrica de Ar Úmido $\quad 76$

5.4.2 Viscosidade do Fluxo de Ar 77 
5.4.3 Vazão Mássica de Ar Úmido $\quad 77$

5.4.4 Massa específica do Ar Ambiente $\quad 77$

5.4.5 Constante do Ar Ambiente $\quad 78$

5.4.6 Umidade Absoluta $\quad 78$

5.4.7 Pressão de Saturação $\quad 79$

5.5 Eficiência Volumétrica $\quad 79$

5.6 Fator Lambida $(\lambda) \quad 79$

5.6.1 Relação Ar/Combustível Real $\quad 80$

5.6.2 Relação Ar/Combustível Estequiométrica $\quad 80$

5.7 Taxa de Substituição $\quad 81$

5.8 Atraso na Combustão $\quad 81$

6. Modelagem do Cálculo do Calor Aparente Liberado 82

6.1 Hipóteses 82

6.2 Primeira Lei da Termodinâmica para Volume de Controle 83

6.3 Razão de Calores Específicos $\quad 85$

6.4 Temperatura no Interior do Cilindro 86

6.5 Volume do Cilindro em uma Determinada Posição $(\theta)$

7. Combustível 89

7.1 Óleo Diesel $\quad 89$

7.1.1 Óleo diesel (S10 e S500) de Uso Rodoviário 89

7.1.2 Óleo diesel (S1800) de Uso Não Rodoviário 90

7.1.3 Óleo diesel marítimo DMA/DMB 90

7.2 Mistura Óleo Diesel/Biodiesel 90

7.3 Etanol 93

8. Resultados e Discussões 96

8.1 Resultados dos Testes com Motor Original - Teste $0 \quad 96$

8.2 Resultados dos Testes com Motor Modificado Operando com Diesel - Teste D 100

8.2.1 Resultados do Teste D1 100

8.2.2 Resultados do Teste D2 101 
8.2.3 Resultados do Teste D3

8.3 Resultado dos Testes com o Motor Modificado Operando com Diesel e Etanol - Teste DE, ED e EDE 103

8.3.1 Resultados do Teste DE 103

8.3.2 Resultados do Teste ED 109

8.3.3 Resultados do Teste EDE 114

9. Conclusões 120

9.1 Comparação dos resultados dos Testes DE, ED e EDE 121

9.1.1 Estratégia de Injeção 122

9.1.2 Taxa de substituição de diesel por etanol 123

9.1.3 Eficiência 124

9.2 Recomendações 124

9.3 Sugestões 125

10. Referências Bibliográficas 130

Anexos

Anexo A - Métodos de Análises Fisicoquímicas $\quad 135$

Anexo A1 - Mistura Diesel/Biodiesel (ASTM) 135

Anexo A2 - Mistura Diesel/Biodiesel (ABNT) 136

Anexo A3 - Mistura Diesel/Biodiesel (EM ISO) 137

Anexo A4 - Etanol (NBR) 138

Anexo A5 - Etanol (D) 139

Anexo A6 - Etanol (E) 140

Anexo A7 - Etanol (ISO) 141

Anexo B - Incerteza na Medição 142 


\section{Lista de figuras}

Figura 1.1: Injeção de combustível no motor RCCl. 26

Figura 3.1: Bancada Experimental do Motor Original 38

Figura 3.2: Bancada Experimental do Motor Modificado 39

Figura 3.3: Motor YANMAR NSB50. 40

Figura 3.4: Conjunto Roda Fônica - Sensor de Posição. 41

Figura 3.5: Sensor de Posição. $\quad 42$

Figura 3.6: Dinamômetro Elétrico. 43

Figura 3.8: Sensor de Torque. 43

Figura 3.9: Inversor de Frequência. $\quad 44$

Figura 3.10: Dissipador de Potência. $\quad 44$

Figura 3.11: Sensor de Pressão. $\quad 45$

Figura 3.12: Corioli CFM010. $\quad 46$

Figura 3.13: Sensor de Fluxo de Ar. $\quad 47$

Figura 3.14: Balança Sartorius PMA7501. 48

Figura 3.15: Sistema de Injeção de Etanol (SIE). 49

Figura 3.16: Bomba de Combustível de Baixa Pressão. 50

Figura 3.17: Manômetro. $\quad 50$

Figura 3.18: Regulador de Pressão. $\quad 50$

Figura 3.19: Bomba de Alta com Unidade de Acionamento. 51

Figura 3.20: Mangueira de Combustível e Acumulador de Pressão. 52

Figura 3.21: Common Rail-Etanol com Sensor de Pressão e Injetor. 52

Figura 3.22: Injetor de Etanol. 53

Figura 3.23: Inversor de Frequência. 53

Figura 3.2: Fonte Estabilizada FTE1310 HOBBY. 54

Figura 3.25: Sistema Lubrificante da Unidade de Acionamento. 55

Figura 3.26: Sistema de Injeção de Diesel.

Figura 3.27: Bomba de Alta Pressão CP3.3 57

Figura 3.28: Common Rail - Diesel. 
Figura 3.29: Cabeçote Modificado.

Figura: 3.30: Tampas do cabeçote: original (A) e modificada (B).

Figura 3.31: Cabeçote adaptado com alojamento para os injetores diesel e etanol (1). Cabeçote adaptado com injetor diesel e etanol (2). Cabeçote adaptado com os injetores 60 diesel e etanol e com a tampa do cabeçote (3).

Figura 3.32: Cabeçote original (A). Cabeçote adaptado (B) com injetor de etanol (1), injetor de diesel (2) e orifício de captação de pressão.

Figura 3.33: Primeira versão do alojamento do injetor diesel (1). Alojamento do injetor de etanol (2). Desgaste na parede da válvula de admissão (3). Fratura (4).

Figura 3.34: Rompimento da parede do alojamento da válvula de admissão (1) do cabeçote do motor. Fissuras na região do furo destinado a receber 0 alojamento do injetor diesel (2). Fissuras no alojamento do parafuso de fixação do cabeçote no motor (3).

Figura 4.1: Tela de controle do perfil dos injetores de diesel e de etanol

Figura 4.2: Tela de controle de pressão nas bombas de alta pressão dos sistemas de injeção de diesel e de etanol.

Figura 6.1: Volume de Controle da Câmara de Combustão

Figura 6.2: Geometria do Motor (adaptado do Heywood, 1988).

Figura 8.1: Resultados do motor original - Teste 0.

Figura 8.2: Calor aparente liberado motor original, operando a 1600 $\mathrm{rpm}$, com vazão de $0,25 \mathrm{~kg} / \mathrm{h}$.

Figura 8.3: Pressão no interior do cilindro do motor original, operando a $1600 \mathrm{rpm}$, com vazão de $0,25 \mathrm{~kg} / \mathrm{h}$.

Figura 8.4: Resultados de energia e de potência dos motores original e modificado obtidos nos experimentos 4,5 e 6 - Teste DE.

Figura 8.5: Rendimento dos motores original e modificado nos experimentos 4, 5 e 6 - Teste DE.

Figura 8.6: Calor aparente liberado dos experimentos 4,5 e 6 do Teste DE.

Figura 8.7: Pressão no cilindro dos experimentos 4, 5 e 6 do Teste DE.

Figura 8.8: Rendimento dos motores original e modificado - Teste ED.

Figura 8.9: Energia total empregada e a potência produzida nos motores original e modificado nos experimentos Teste ED. 
Figura 8.10: Calor aparente liberado - Teste ED.

Figura 8.11: Pressão no interior do cilindro - Teste ED.

Figura 8.12: Rendimento e potência dos motores original e modificado - Teste EDE

Figura 8.13: Energia total empregada e a potência produzida nos motores original e modificado nos experimentos Teste EDE.

Figura 8.14 - Calor aparente liberado - Teste EDE.

Figura 8.15: Pressão no interior do cilindro-Teste EDE.

Figura 9.1: Calor aparente do Teste DE, ED e EDE com vazão de diesel de $0,15 \mathrm{~kg} / \mathrm{h}$ e a vazão total de etanol de 0,43 $\mathrm{kg} / \mathrm{h}$.

Figura 9.2: Pressão no interior do cilindro do Teste DE, ED e EDE com vazão de diesel de $0,15 \mathrm{~kg} / \mathrm{h}$ e a vazão total de etanol de $0,43 \mathrm{~kg} / \mathrm{h}$.

Figura 9.3: Pressão no interior do cilindro do motor modificado funcionando com etanol (E-170) e sem a injeção de combustível (sem combustão).

Figura 9.4: Pressão no interior do cilindro do motor modificado funcionando com etanol (E+2/E-170) e sem a injeção de combustível (sem combustão). 


\section{Lista de tabelas}

Tabela 2.1 - Limites das Emissões - PROCONVE 30

Tabela 3.1 - Dados Técnicos do Motor YANMAR NSB50 41

Tabela 3.2 - Dados Técnicos do Inversor de Frequência 44

Tabela 3.3 - Dados Técnicos do Motor Elétrico do Dinamômetro 45

Tabela 3.4 - Dados Técnicos do Medidor de Pressão KISTLER 6052C 45

Tabela 3.5 - Dados Técnicos do Corioli 46

Tabela 3.6 - Dados Técnicos do Medidor de Fluxo 47

Tabela 3.7 - Dados Técnicos do Transdutor de Pressão 47

Tabela 3.8 - Dados Técnicos da Balança Sartorius PMA7501 48

Tabela 3.9 - Dados Técnicos do Injetor de Etanol 53

Tabela 3.10 - Dados Técnicos do Motor Elétrico (SIE) 54

Tabela 3.11 - Dados Técnicos do Injetor de Diesel 58

Tabela 3.12 - Dados Técnicos do Motor Elétrico (SID) 58

Tabela 4.1: Mapeamento do Motor Original 66

Tabela 4.2: Teste D1 68

Tabela 4.3: Teste D2 69

Tabela 4.4: Teste D3 69

Tabela 4.4: Estratégia de Injeção 72

Tabela 7.1 - Especificação do óleo diesel BX a B30. 91

Tabela 7.2: Especificação do Etanol.

Tabela 8.1: Resultados dos Testes com Motor Original - Teste $0 \quad 97$

Tabela 8.2: Resultados do Teste D1 (Motor Modificado) 100

Tabela 8.3: Extrato dos resultados do Teste 0 (Motor Original) 100

Tabela 8.4: Resultados do Teste D2 101

Tabela 8.5: Resultados do Teste D3 102

Tabela 8.6: Resultados do Teste DE (Motor Modificado) 104

Tabela 8.7: Extrato 1 dos Resultados do Teste 0 (Motor Original) 104

Tabela 8.8 Resultados do Teste ED (Motor Modificado) 110

Tabela 8.9 Extrato 2 dos Resultados do Teste 0 (Motor Original) 110

Tabela 8.10 Extratos dos Resultados do Teste ED (Motor Modificado) 112

Tabela 8.11 Resultados do Teste EDE (Motor Modificado) 115 
Tabela 8.12 Extrato 3 dos Resultados do Teste 0 (Motor Original) 115

Tabela 9.1 Resultado da injeção de etanol a -170 $\cong$ PMS 126

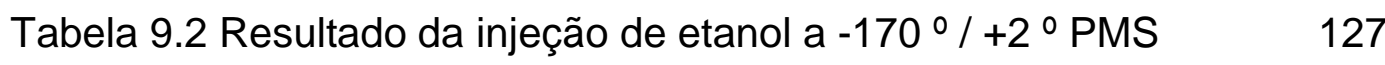




\section{Lista de símbolos}

ANP Agência Nacional do Petróleo, Gás Natural e Biocombustíveis

$\begin{array}{ll}\text { ATAC } & \text { Active Thermo Atmosphere Combustion } \\ a & \text { Raio do Virabrequim } \\ B & \text { Diâmetro do Cilindro } \\ \text { B20 } & 20 \% \text { de biodiesel e } 80 \% \text { de diesel } \\ \mathrm{C} & \text { Carbono, Combustível } \\ \mathrm{CO} & \text { Monóxido de Carbono } \\ \mathrm{CO}_{2} & \text { Dióxido de Carbono }\end{array}$

CONAMA Conselho Nacional do Meio Ambiente

$c_{p} \quad$ Calor Específico à Pressão Constante

$c_{v}$

Calor Específico a Volume Constante

$\mathrm{D}$

Diesel

DI

Direct Injection

$\frac{d Q_{n}}{d t}$

Taxa de Calor Liberado

$\mathrm{E}$

Etanol

EGR

Recirculação dos Gases de Escape

E20

$20 \%$ de etanol e $80 \%$ de gasolina

E85

$85 \%$ de etanol e $15 \%$ de gasolina

EPA

Enviroment Protection Agency

EURO Comissão Europeia para veículos leves e pesados

FTP

Federal Test Procedure

$\mathrm{HC}$

Hidrocarbonetos

$\mathrm{HCCl} \quad H o m o g e n e o u s$ Charge Compression Ignition

ICO Ignição por compressão

ICE Ignição por centelha

L Comprimento da Biela

LTC Low Temperature Combustion

MP Material Particulado 


$\begin{array}{ll}\text { MCR } & \text { Máquina de Compressão Rápida } \\ \mathrm{ms} & \text { milissegundos } \\ \mathrm{NOx} & \text { Óxidos de Nitrogênios } \\ \mathrm{p} & \text { Pressão } \\ P_{\theta} & \text { Pressão no interior do cilindro } \\ P_{0} & \text { Pressão no momento da admissão do ar } \\ \mathrm{PCCl} & \text { Premixed Charge Compression Ignition } \\ \mathrm{PFI} & \text { Port Fuel Injection } \\ \mathrm{PMI} & \text { Ponto Morto Inferior } \\ \mathrm{PMS} & \text { Ponto Morto Superior } \\ \mathrm{PROCONVE} & \text { Programa de Controle da Poluição do Ar por Veículos } \\ \mathrm{R} & \text { Constante Universal dos Gases } \\ \mathrm{RCCl} & \text { Reactivity Controlled Compression Ignition } \\ \mathrm{SID} & \text { Sistema de Injeção de Diesel } \\ \mathrm{SIE} & \text { Sistema de Injeção de Etanol } \\ r_{C} & \text { Razão de Compressão } \\ \mathrm{S} & \text { Curso Total do Pistão } \\ \mathrm{S}_{\theta} & \text { Curso do pistão } \\ \text { SOx } & \text { Óxidos de Enxofre } \\ T_{\theta} & \text { Temperatura no interior do cilindro } \\ T_{0} & \text { Temperatura no momento da admissão do ar } \\ \text { US } & \text { United States } \\ V_{\theta} & \text { Volume no interior do cilindro } \\ V_{0} & \text { Volume com válvula de admissão fechada } \\ \mathrm{V}_{\mathrm{D}} & \text { Volume Deslocado } \\ \mathrm{V}_{\mathrm{M}} & \text { Volume Morto } \\ \mathrm{V} \mathrm{T} & \text { Volume Total } \\ & \end{array}$




\section{Lista de símbolos gregos}

$\gamma \quad$ Razão entre os Calores Específicos

$\theta \quad$ Ângulo do virabrequim 